\title{
Synthesis And Anti-Inflammatory Activity On Sulpha/Substituted Pyrazoles(1,2-Diazole)
}

\author{
${ }^{1,}$ Rigvendra Malik , ${ }^{2, N a r e s h ~ P a l, ~}{ }^{3,}$ Gajendra Singh and ${ }^{4,}$ C.P. singh \\ ${ }^{1,2}$ Dept. of Chemistry, S.S.V. (P.G) College, Hapur (U.P.) \\ ${ }^{3,4}$ Dept. of Chemistry, Research Division, Sahu Jain (P.G.) College, Najibabad-246763 (U.P.)
}

\begin{abstract}
A Novel compound namely $N^{l}$-(Nicotinoyl) 3,5 dimethyl-4-( $N^{l}$-4-sulfamoylazo)-1,2-diazole has been synthesised by two step processes. Synthesis of $N^{l}$-4-sulphamoylphenylhydrazono-3,5-dimethyl propane1,3-dione and sulphonamide, which interacting with Nicotinic acid hydrazide to form final compound. The newely synthesised compound $N^{I}$-(nicotinoyl)-3,5-dimethyl 4-(N-4-sulfamoyl phenyl azo) 1,2-diazoles was screened for anti-inflammatory activity.
\end{abstract}

KEYWORDS: synthesis, anti-inflammatory activity, sulphonamide 1,3-diketone, Nicotinic acid hydrazide.

\section{INTRODUCTION}

A heterocyclic compound is one which possess a cyclic structures with atleast one hetero atom in the ring. Nitrogen oxygen and sulphur are the most common hetero atoms. Heterocyclic compounds are very widely distributed in nature and essential to the life in various ways. Vitamin C exist in the form of five membered (furan) or six membered (Pyran) rings containing one oxygen atom. Most member of Vitamin B group possess heterocyclic ring containing nitrogen. One example is vitamin $\mathrm{B}_{6}$ (Pyridoxine), which derivative of pyridine essential in amino acid metabolism. 1,2-diazole is a heterocyclic compound having varied biological activity and still of great scientific interest now a days. They are widely found in bioorganic and medicinal chemistry with application in drug discovery. Nitrogen based heterocyclic compounds are very important in the field of medicinal chemistry. The present diazoles were prepared because of its good biological activity. Compounds including a 1,2-diazole nucleus and $\mathrm{N}$-substituted derivatives are known to possess various biological activity[1]. Among these types of molecules have been shown to have various important biological activity such as antibacterial, antifungal, antiviral, diuretic, antituberculostatic, anti-HIV, antihistaminic anticancer, anticonvulsant, anti-inflammatory and analgesic properties[3-7].

Sulpha/substituted 1,2-diazoles may serve as the alternative sources for the development of new antiinflamatory agents due to their biological activity. Sulpha/substituted 1,2-diazoles used for the treatment of anti-inflammatory in different systems of medicine have shown diuretic activity when tested on animal models. On the basis of the use of diuretics, but no previous pharmacological study was carried out to test antiinflammatory the activity of sulpha/substituted 1,2-diazoles. The main aim of the present investigation was to evaluate the claimed anti-inflammatory activity of sulpha/substituted 1,2-diazoles.

\section{MATERIAL AND METHOD}

The 1.3-diketones, sulphanilamide, nicotinic acid hydrazide and all reference compound were purchased from Aldrich Chemicals, Ethanol, sodium acetate, glacial acetic acid and all other reagents were purchased from S.D. Chem. TLC was performed on pre-coated plastic sheets of silica gel G/UV-254 of $0.2 \mathrm{~mm}$ thickness (Macherey-Nagel, Germany).

\subsection{General}

Melting points of the ( $\mathrm{N}^{1}$-nicotinoyl) 3,5 dimethyl-4( $\mathrm{N}^{1}-4$-sulfamoylphenylazo)-1,2-diazole was determined using an open-ended capillary tube method and are uncorrected. The purity of the synthesized compound was checked by TLC. A FT-IR spectrum was recorded on a Perkin-Elmer 1605 series FT-IR in a $\mathrm{KBr}$ Disc, ${ }^{1} \mathrm{H}$ NMR spectra was recorded at $300 \mathrm{MHz}$ on a Burker FT-NMR spectrophotometer using TMS as internal standard.

Step-1

Synthesis of $N^{1}$-4-sulphamoylphenyl hydrazono-3,5-dimethyl propane-1,3-dione

An ice cooled solution of 3,5-dimethyl propane-1,3-dione (0.03 mole) in ethanol containing sodium acetate (6 grams) a diazotized solution of sulphonamide $(0.05$ mole) were gradually added with stirring and 
cooling. The reaction mixture was further strirring for 20 minutes, the coloured hydrazono compounds precipitated by addition of ice cold water. It was filtered off, washed with water, dried and recrystallised from ethanol/acetic acid [Fig. 1]. On analysis, it was found to be $\mathrm{N}^{1}$-4-sulphamoylphenyl hydrazono-3,5-dimethyl propane-1,3-dione [Fig. 1].

\section{$N^{I}$-4-sulphamoylphenyl hydrazono 3,5-dimethyl propane-1,3-dione}

A yellow crystalline powder. mp $198-200^{\circ} \mathrm{C}$, Yield $82.34 \%$, molecular formula $\mathrm{C}_{16} \mathrm{H}_{15} \mathrm{O}_{4} \mathrm{~N}_{3} \mathrm{~S}$ (348.76): C, 55.10; H, 4.34; O, 18.35; N, 12.04; S, 10.17. Found: 54.92; 4.56; O, 18.17; N, 12.48; S, 9.87, IR (KBr) in cm ${ }^{1} 1440(\mathrm{C}-\mathrm{C}), 1560\left(\mathrm{C}=\mathrm{C}\right.$ of aromatic ring), $1260(\mathrm{C}-\mathrm{N}), 1680(\mathrm{C}=\mathrm{O}), 3087(\mathrm{NH}), 3275\left(\mathrm{SO}_{2} \mathrm{NH}_{2}\right) .{ }^{1} \mathrm{HNMR}$ $\left(\mathrm{CDCl}_{3}\right) \delta$ in ppm, 2.81 (s, $\left.3 \mathrm{H} \mathrm{C}_{\underline{3}}\right), 6.75-7.68(\mathrm{~m}, 9 \mathrm{H}, \mathrm{Ar}-\mathrm{H}), 6.92\left(\mathrm{~s}, 2 \mathrm{H} \mathrm{N}_{\underline{2}}\right), 10.43(\mathrm{~s}, 1 \mathrm{H} \mathrm{N} \underline{\mathrm{H}})$.

\section{Step-II}

Synthesis of $N^{1}$-(nicotinoyl, 3,5-dimethyl-4(N-4-sulfamoyl phenylazo)-1,2-diazole

A solution of $\mathrm{N}^{1}$-4-sulphamoylphenyl-hydrazono 3,5-dimethyl propane-1,3-dione $(0.02 \mathrm{~mole})$ in glacial acetic acid was added to Nicotinic acid hydrazide $(0.05$ mole) refluxed on water bath for 6 hours and left overnight. On cooling, shining recrystallised crystals, separated out which was collected by filtration, washed well with water, dried and recrystallised from glacial acetic acid to give $\mathrm{N}^{1}$-(nicotinoyl)3,5 dimethyl-4(N-4sulfamoylphenylazo)-1,2-diazole [Fig. 2].

\section{$N^{l}$ (Nicotimolyl)-3,5-dimethyl-4(N-sulfamoylphenylazo)-1,2-diazole}

A yellow crystalline powder, mp 226-228 ${ }^{\circ} \mathrm{C}$, Yield $72.13 \%$. molecular formula $\mathrm{C}_{23} \mathrm{H}_{20} \mathrm{O}_{3} \mathrm{~N}_{6} \mathrm{~S}$, anal. Calcd for $\mathrm{C}_{23} \mathrm{H}_{20} \mathrm{O}_{3} \mathrm{~N}_{6} \mathrm{~S}$ (463.90: C, 59.55; H, 4.34; O, 10.35; N, 18.12; S, 18.12; S, 7.64. Found: C, 58.97; H, 4.64; O, 10.29; N, 18.37; S, 7.73. IR (KBr) in $\mathrm{cm}^{-1} 740(\mathrm{C}-\mathrm{C}), 1240(\mathrm{C}-\mathrm{N}), 1535$ (C=C of aromatic ring), 1585 $(\mathrm{C}=\mathrm{N}), 1460(\mathrm{~N}=\mathrm{N}), 3055($ aromatic $\mathrm{C}-\mathrm{H}), 3135(\mathrm{NH}), 1707(\mathrm{C}=\mathrm{O}), 3082\left(\mathrm{NH}_{2}\right) .{ }^{1} \mathrm{HNMR}\left(\mathrm{CDCl}_{3}\right) \delta$ in ppm,

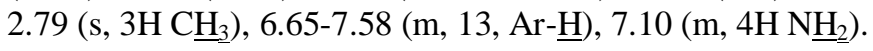

\section{Animals}

Adult's male Wistar albino rats, each in the weight range of 180-200 gm were used for this experiment. They were procured from National Vertinary Research centre, Bareilly, India. The animals were randomly allocated to six treatment groups of six animals each and kept in polypropylene cages and housed under standard conditions of temperature, humidity, dark light cycle (12h-12h) and diet.

\section{Anti-inflammatory activity ${ }^{8,9}$}

The activity of the newly synthesized compounds compared to indomethacin as a reference compound was measured before and $4 \mathrm{~h}$ after carrageenan injection. Percent of the oedema inhibition was calculated as regards saline control group and potency was calculated as regards the percentage of the change of indometacin and tested compounds [24], as depicted in Table 2. All the tested compounds showed a reasonable inhibition of oedema size ranging between a $13.5 \%$, for compound $25.4 \%$ for compound (b) $28 \%$ for compound, $32.7 \%$ for compound sulphonamide 1,2-diazole (c), $44.4 \%$ for compound sulphapyrimidine 1,2-diazole (d) and $29.9 \%$ for indomethacin. In activity relationship point of view, the anti-inflammatory activity of the pyrimidine was found to be the promising one. But sulphonamide also showed good anti-inflammatory activity (32.7\%).

Table 1. Anti-inflammatory effect of sulpha/substituted-1,2-diazoles

\begin{tabular}{|c|c|c|c|c|c|c|}
\hline \multirow[t]{2}{*}{ Compound } & \multirow{2}{*}{$\begin{array}{c}\text { Dose } \\
(\mathrm{mg} / \mathrm{kg})\end{array}$} & \multicolumn{2}{|c|}{ Oedema } & \multirow{2}{*}{$\begin{array}{l}\text { Oedema }(\%) \\
(\mathrm{X} \pm \mathrm{SE})\end{array}$} & \multirow{2}{*}{$\begin{array}{c}\text { Oedema } \\
\text { inhibition }(\%)\end{array}$} & \multirow[t]{2}{*}{ Potency } \\
\hline & & $\begin{array}{l}\text { Zero min } \\
\text { (basal) }\end{array}$ & $\begin{array}{c}4 \mathrm{~h} \text { oedema }(\mathrm{cm})(\% \\
\text { increase })\end{array}$ & & & \\
\hline Control & $1 \mathrm{~mL}$ saline & $0.23 \pm 0.006$ & $0.46 \pm 0.01$ & $109.1 \pm 6.3$ & -- & -- \\
\hline $\mathrm{a} \mathrm{C}_{14} \mathrm{H}_{10} \mathrm{NCl}$ & 70 & $0.20 \pm 0.002$ & $0.37 \pm 0.01$ & $81.3 \pm 4.8$ & -25.48 & 0.9 \\
\hline $\mathrm{b} \mathrm{C}_{14} \mathrm{H}_{10} \mathrm{NF}$ & 70 & $0.22 \pm 0.002$ & $0.38 \pm 0.03$ & $78.3 \pm 4.1$ & -28 & 0.9 \\
\hline c $\mathrm{C}_{14} \mathrm{H}_{10} \mathrm{~N}_{2} \mathrm{O}_{2}$ & 70 & $0.21 \pm 0.003$ & $0.41 \pm 0.2$ & $94.8 \pm 5.3$ & -13.1 & 0.4 \\
\hline $\mathrm{d} \mathrm{C}_{20} \mathrm{H}_{16} \mathrm{~N}_{2} \mathrm{O}_{2} \mathrm{~S}$ & 70 & $0.22 \pm 0.002$ & $0.37 \pm 0.02$ & $73.4 \pm 4.4$ & -32.7 & 1.1 \\
\hline $\mathrm{e}_{24} \mathrm{H}_{16} \mathrm{~N}_{4} \mathrm{O}_{2} \mathrm{~S}$ & 70 & $0.20 \pm 0.00$ & $0.32 \pm 0.001$ & $60.7 \pm 4.9$ & -44.4 & 1.5 \\
\hline $\mathrm{f} \mathrm{C}_{24} \mathrm{H}_{18} \mathrm{~N}_{4} \mathrm{O}_{2} \mathrm{~S}$ & 70 & $0.20 \pm 0.00$ & $0.32 \pm 0.004$ & $60.7 \pm 4.9$ & -44.4 & 1.5 \\
\hline Indomethacin & 35 & $0.26 \pm 0.003$ & $0.39 \pm 0.03$ & $76.5 \pm 3.6$ & -29.9 & 1 \\
\hline
\end{tabular}

\section{CONCLUSION}

The present study that, synthesised compound $\mathrm{N}^{1}$ (Nicotimoyl)-3,5-dimethyl 4-sulfamoylphenylazo)1,2-diazole possess significant at 100 and $200 \mathrm{mg} / \mathrm{kg}$ but the effect declined at higher dose. 


\section{ACKNOWLEDGMENT}

The authors express their sincere thank to principal, S.S.V. (P.G.) College, Hapur for providing lab facilities and also thank to CDRI Lucknow, IIT, Roorkee for Providing IR, NMR spectral data and pharmacological data.

\section{REFERENCES}

[1] Achson a, An introduction to the chemistry of heterocyclic compounds $3^{\text {rd }}$ edition, Wiley interscience, India 2000.

[2] G. capan, N. Ulusoy, N. Ergenc, M. Krtaz, New 6-phenylimidazo[2,1-b]thiazole derivatives: Synthesis and antifungal activity. Monatsh. Chem. 1999, 130, 1399

[3] R.U. Roy, a. R. Desai, K.R. Desai, "synthesis and Antimicrobial Activity of 1,2,4-Thiazoles, E-Journal of Chemistry, 2005, 2(6), 1 .

[4] M.G. Vigorita, R. Ottana, F. Monforte, R. maccari, A. Trovato, M. T. Monforte, M.F. Taviano, Synthesis and anti-inflammatory, analgesic activity of 3,3-(1,2-ethandiyl)-bis[2-aryl-4-thiazolidinone] chiral compounds. Part 10. Bioorg. Med. Chem. 2001, 11, 2791.

[5] C.V. Kavitha, S. Basappa, S. Nanjunda, K. Mantelingu, S. Doreswamy, M. A. Sridhar, J.S. Prasad, K.S. Rangappa, Synthesis of new bioactive venlafaxine analogs: Novel thiazolidin-4-ones as antimicrobilas. Bioorg. Med. Chem. 2006, 14, 2290.

[6] R. Ottana, R. Maccari, M.L. Barreca, G.Bruno, A. Rotondo, A. Rossi, G. Chiricosta, R. Di Paola, L. Sautebin, S. Cuzzocrea, M.G. Vigorita, 5-Arylidene-2-imino-4-thiazolidinones: Design and synthesis of novel anti-inflammatory agents. Bioorg. Med. Chem. 2005, 13, 4243.

[7] G. kucukguzel, A. Kocatepe, E. De Clercq, F. Sahin, M. Gulluce, Synthesis and biological activity of 4-thiazolidinones, thiosemicarbazides derived from diflunisal hydrazide. Eur. J. med. Chem. 2006, 41, 353.

[8] Radwan, M.A.A., Rageb E.A. Saby, N.M. EI-Shenaway, S.M., Potential anti-inflammatory and analgesic agents, Bioorganic and medicianal chemistry 2007, 15, pp 3832-3841.

[9] Singh N, Bhatia, S.K., Kumar A, Thiazolyl/formazanyl-1,2-diazoles as potent anti-inflammatory agent, European Jour. Of medicinal Chem., 2008, 43, 2597-2609.

\section{Synthesis of novel $N^{I}$-(Nicotinoyl)-3,5-dimethyl-4(N-4-sulfamoylphenylazo)-1,2-diazole}<smiles>CNC(C)=O</smiles>

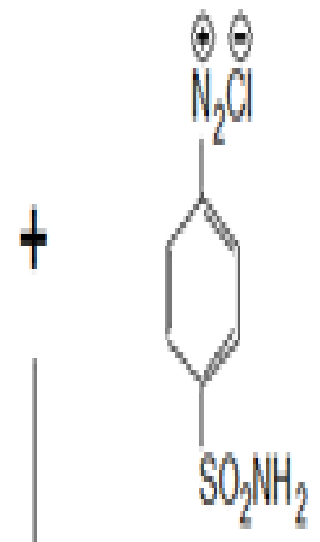

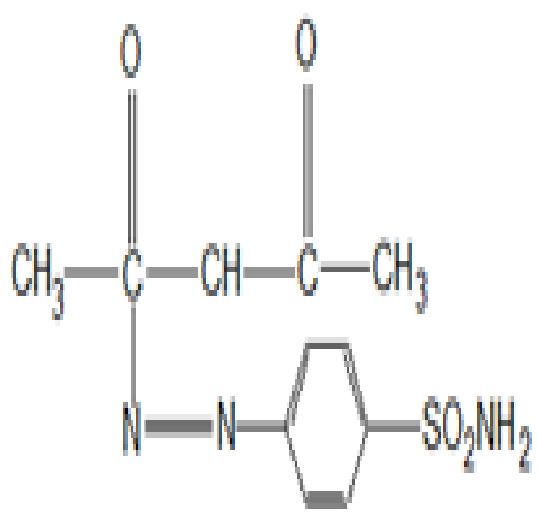

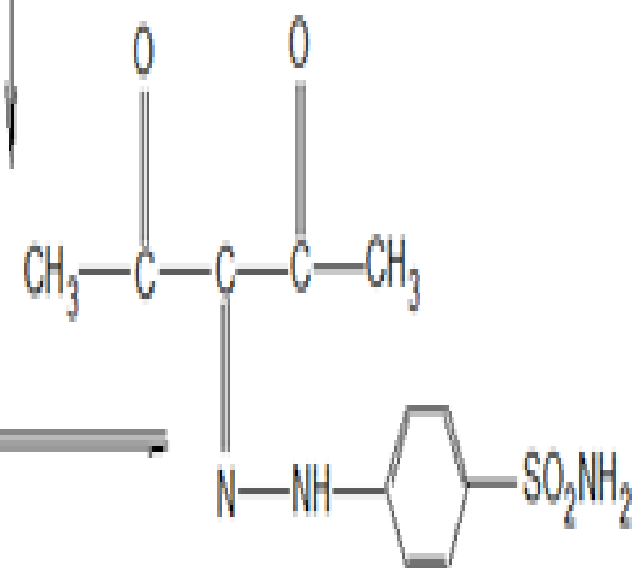

Fig.1 Synthesis of $\mathbf{N}^{1}$-4-sulphamoylphenyl hydrazono-3,5-dimethyl propane-1,3-dione 
<smiles></smiles><smiles>NC(=O)c1cccnc1</smiles>

Glacial acetic acid<smiles></smiles><smiles>Nc1ccc(S(N)(=O)=O)cc1</smiles><smiles>CC(=O)C(=NNC(=O)c1cccnc1)C(C)=O</smiles><smiles>N#CC1CCC(S(N)(=O)=O)CC1</smiles><smiles>Cc1nn(C(=O)c2cccnc2)c(C)c1N=N</smiles>

Fig.2 Synthesis of $\mathrm{N}^{\mathbf{1}}$-3,5-dimethyl-4(N-4-sulphamoylphenylazo)-1,2-diazole 